\title{
Regionale Identität und Kontextualisierung
}

\author{
Stadtansichten auf Titelblättern von Bibeln
}

Historische Ortsansichten finden sich nicht nur in den Graphischen Sammlungen, sondern auch als Illustrationen alter Drucke. Inhaltliche Aussageabsichten für das Gesamtwerk sind vor allem dort zu vermuten, wo Bilder von Städten an besonders exponierten Stellen wie Titelblättern verwendet werden. Eine interessante Untergruppe stellen dabei Bibelausgaben dar, weil reale Städte als Motiv anders als bei Geographika und Reiseliteratur von den Adressaten nicht erwartet werden. Vor allem im späten 17. und im 18. Jahrhundert begegnen uns Ortsansichten als ein Teil der Gesamtkomposition der einführenden Blätter des Bibeldrucks. Einige Aspekte der Motivation lassen sich beobachten bzw. erschließen.

Bei Stadtansichten geht es um konkrete Haftpunkte der regionalen Identität. Es kommt zu einem Wiedererkennungseffekt: Die vertraute Umgebung, aber auch die Relevanz des eigenen Ortes wird vor Augen geführt. Insbesondere in freien Reichsstädten, Universitätsstädten oder wohlhabenden Handelsstädten stellt der Ort den primären Referenzpunkt dar. Dynastische Loyalitäten zu Herrscherhäusern treten etwas zurück. Städte können aber auch als Obrigkeit fungieren.

Theologisch wird das Hier und Jetzt des in der Bibel bezeugten Heilsgeschehens betont, die individuelle Manifestation der Heilsgeschichte. Der Druckort erscheint als Ort der Vergegenwärtigung und Vermittlung der biblischen Botschaft.

Auf den Einfluss von Pietismus und Aufklärung könnte zurückgehen, dass das Praktische, Empirische, Reale, Alltägliche, Nahe wichtiger wird, auch das dem Individuum eher Verfügbare.

Problem jeder Anknüpfung an geographische, historische oder soziale Gegebenheiten der Adressaten ist, dass sie in anderen Kontexten und nach Änderung der Strukturen die Verstehbarkeit der Inhalte erschwert und schnell veraltet. Eine Vorstufe zeigt sich in der Gestalt von Wappen. Diese symbolisieren die Region des Druckortes bzw. des Auftraggebers in konzentrierter Form, wobei die Herrscherfamilie mit dem betreffenden Gebiet identifiziert wurde. Beliebt war die Kombination des Wappens mit Elementen eines Säulenportals, das den Tempel in Jerusalem bzw. eine Kirche symbolisieren soll. So weist eine Teilausgabe von "Jesus Syrach zu Wittemberg verdeudscht" ein sächsisches Wappen auf (B deutsch 1533 01). Diese Bibelausgabe will - so deutet das Titelblatt an - den Eintritt in den Bereich des Heiligen ermöglichen, tut dies aber als ein sächsischer Druck.

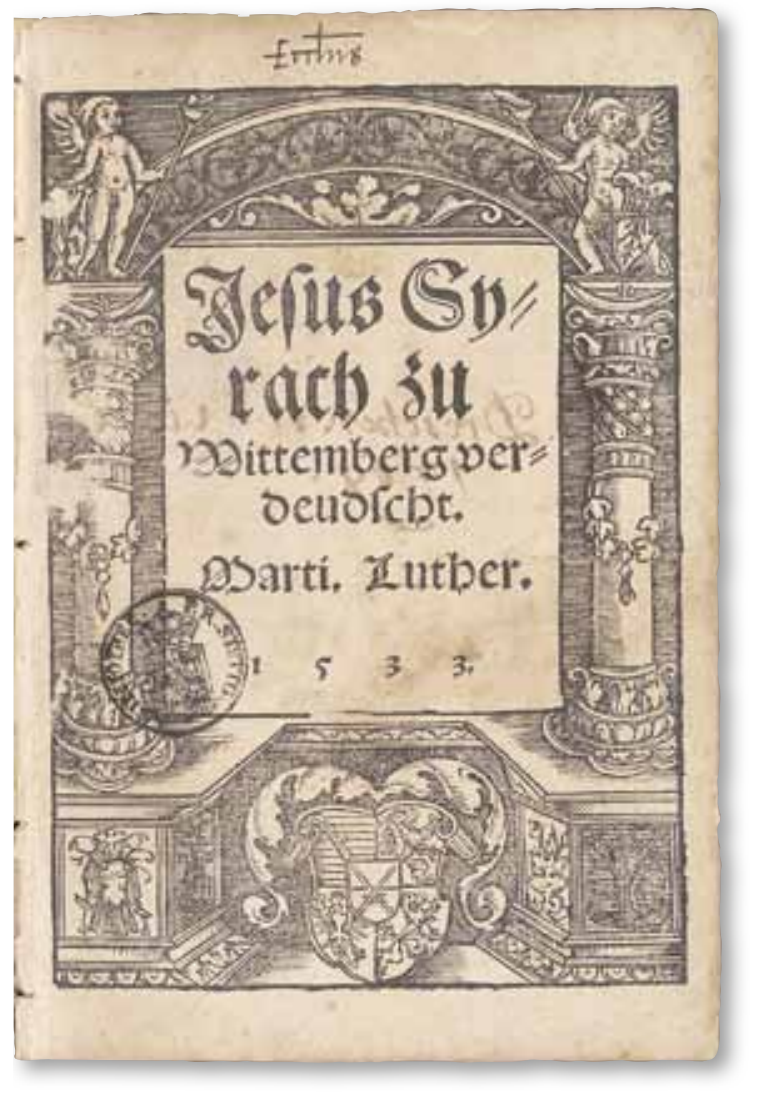

Abb. 1: B deutsch 153301

Aus inhaltlichen Gründen wurde in einigen Ausgaben bewusst auf eine regionale Konkretion verzichtet. So wählt eine gelehrte, griechisch-deutsche Edition des Neuen Testaments als Bildmotiv eine Weltkugel statt eines bestimmten Ortes (B griech. 1730 03) - darauf die aufgeschlagene Bibel, ein Schlüssel sowie ringsherum personifizierte Wissenschaften und ein Bildnis des Herausgebers. Dies bedeutet: Das Neue Testament bietet einen 
wesentlichen Verstehensschlüssel für den Kosmos der Wissenschaften.

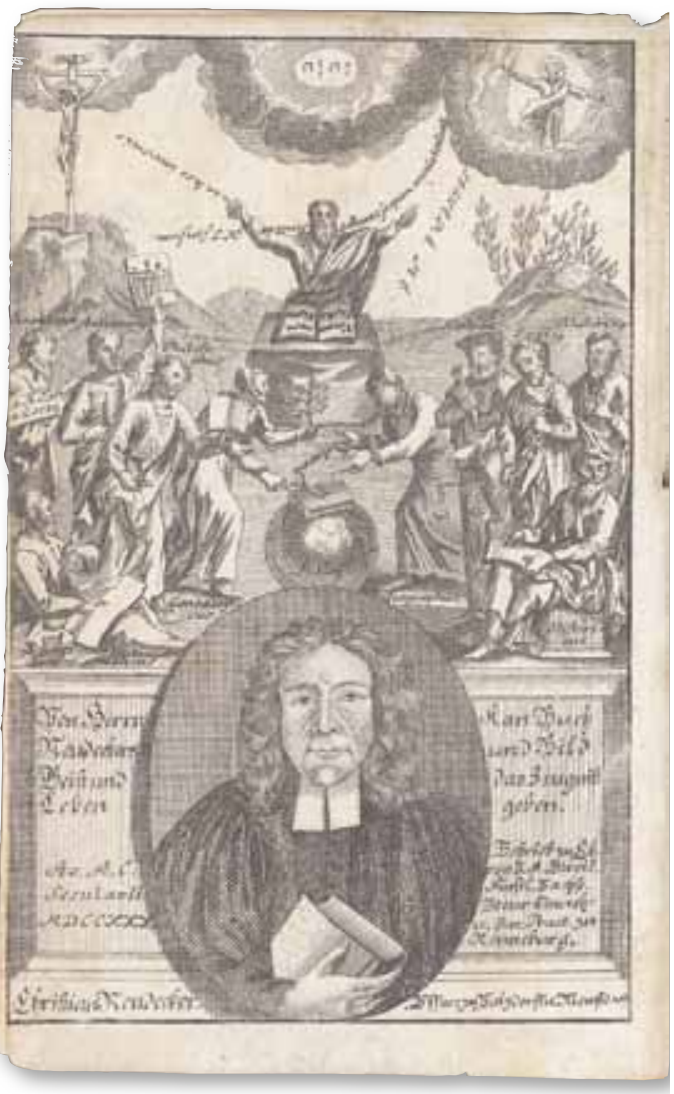

Abb. 2: B griech. 173003

Oder bei der auf dem Titelblatt sichtbaren Stadt handelt es sich um das himmlische Jerusalem, das für das Ende der Zeiten erwartet wird. Der Betrachter sieht sich angesichts seiner Lebensverhältnisse mit einer erhofften Gegenwirklichkeit konfrontiert (Ba deutsch 1743 02).

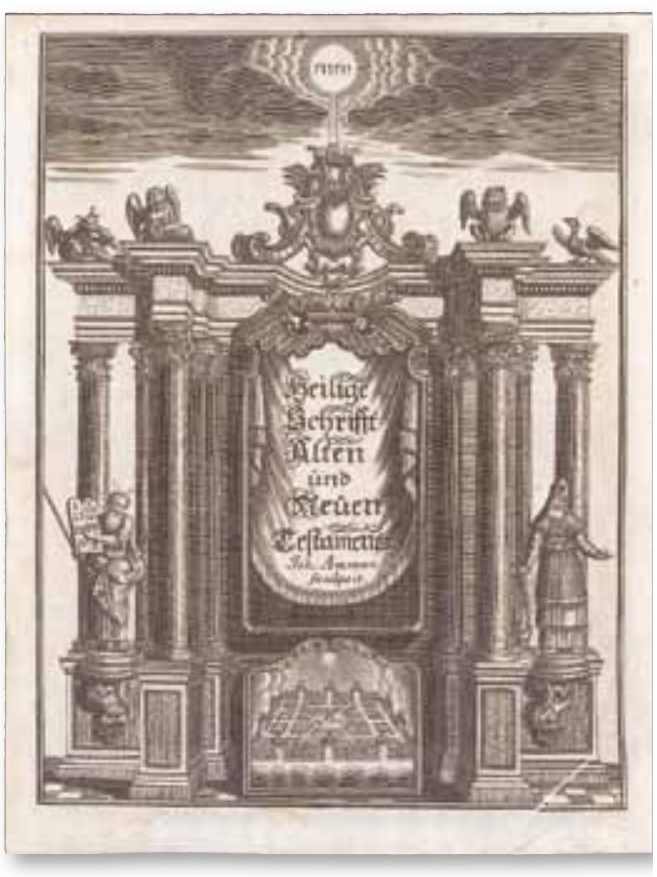

Abb. 3: Ba deutsch 174302
Die aus mystisch-separatistischen Kreisen stammende Berleburger Bibel (Bb deutsch 1726 01) bringt mit ihrer Titelblattillustration Distanz nicht nur zur Welt, sondern auch zur vorfindlichen Kirche zum Ausdruck. Nicht das künftige himmlische Jerusalem, sondern die philadelphische Idealgemeinde (Offenbarung 3,7-11) ist das Leitbild gegenwärtiger kirchlicher Praxis. Dementsprechend verbietet sich jegliche Anknüpfung an bestehende Orte.

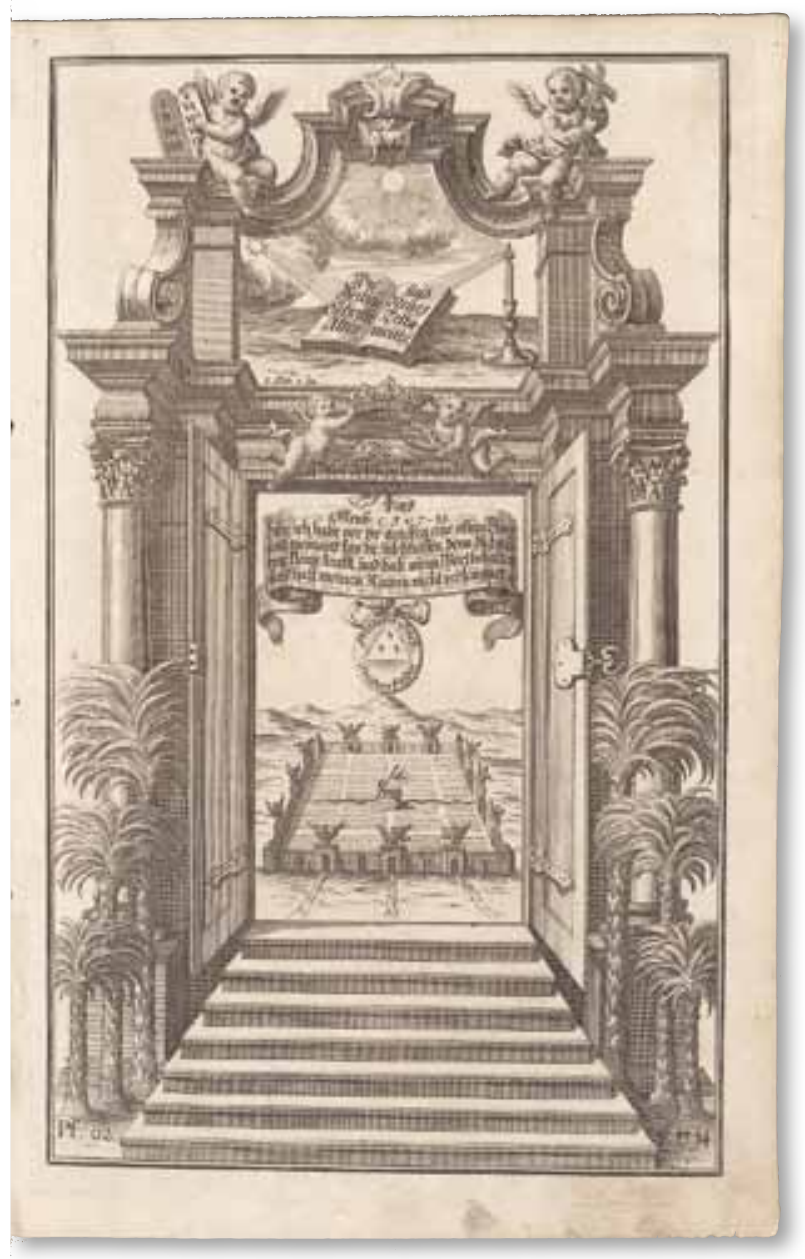

Abb. 4: Bb deutsch 172601

Titelbilder wirken als Korrektiv zur gegenwärtigen Wirklichkeit aber auch dann, wenn sie auf konkrete Zeitumstände Bezug nehmen. Das ist z.B. bei einer 1635 in Lüneburg gedruckten Bibel der Fall (Ba deutsch 1635 01). Bilder und deren Über- und Unterschriften deuten den Kontext des Dreißigjährigen Krieges an. Eine Stadt wird belagert, feindliche Heere treffen aufeinander. Bei der belagerten Stadt ist an eine eher generische Verwendung als Platzhalter für viele Orte zu denken, denen dies geschah. Konkret hingegen ist das historische Ereignis. Szenen aus der Geschichte Israels (Wolken- und Feuersäule, Goldenes Kalb) sowie die gegensätzlichen Wahlsprüche „Germania desola- 
ta" und "Germania resurgens" (verödetes / wieder aufstehendes Deutschland) deuten an, worin der Künstler die Hoffnung auf Besserung begründet sieht. Die Orientierung am Willen Gottes, wie er in der - gedruckt vorliegenden - Bibel zu lesen ist, würde die Menschen zur Umkehr führen.

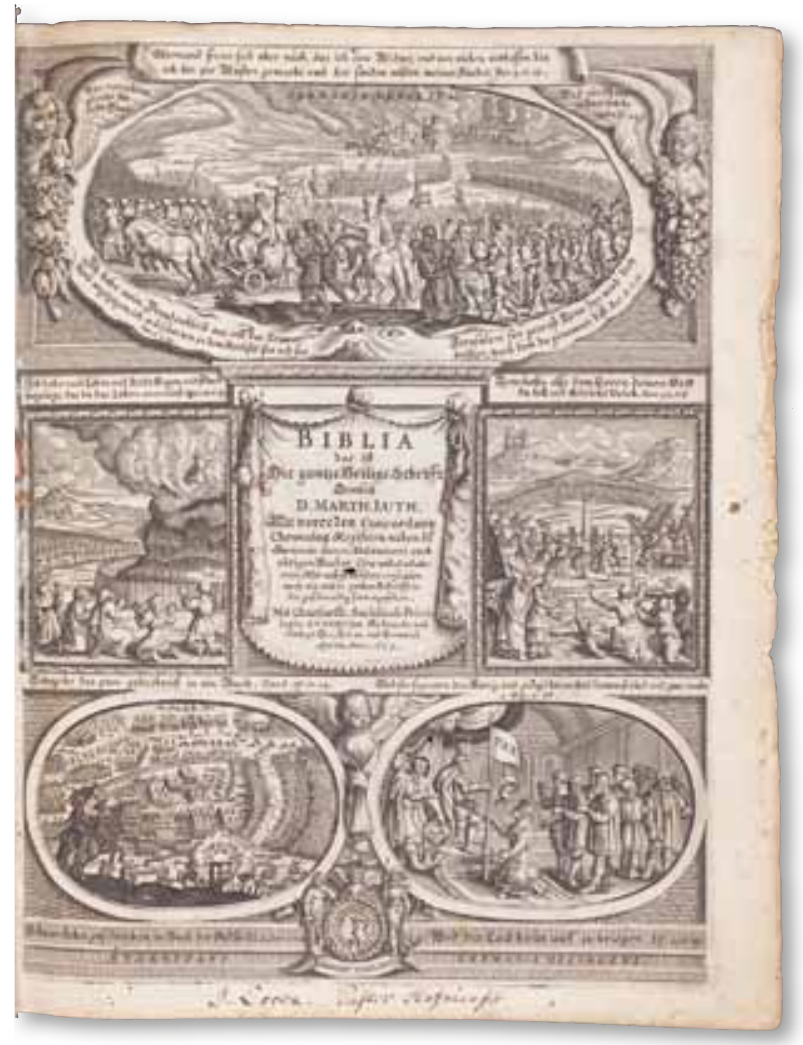

Abb. 5: Ba deutsch 163501

Auf die Zerstörungen des Dreißigjährigen Krieges spielt auch eine Lüneburger Lutherbibel von 1646/1647 an (B deutsch 1646 02). Das Titelkupferbild bezieht die Klage Jesu über das nicht zur Umkehr bereite Jerusalem (Matthäus 23,37) auf eine der Architektur nach charakteristische deutsche Stadt - die gerade geplündert wird! Ein kleines Bild auf dem Titelblatt zeigt eine Schlachtenszene mit der Bitte um Frieden ("Da pacem patriae"). Mose mit den Gesetzestafeln und David mit der Harfe deuten den Ausweg aus der Krise an: Buße bzw. Gotteslob.
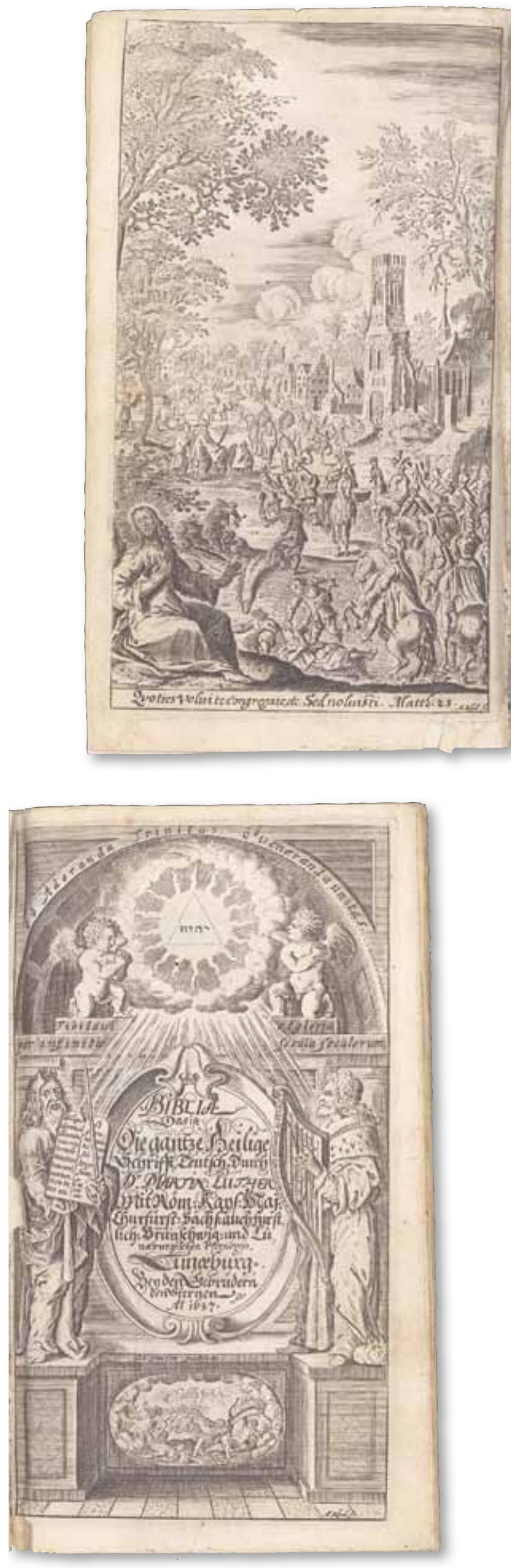

Abb. 6-7: B deutsch 164602 
Umgekehrt gibt es Bibeln, die eine reale Stadt konkret abbilden, jedoch mit den übrigen Illustrationen eher generisch eine allgemeine Situation der Menschen andeuten. Das gilt etwa für eine katholische Erfurter Bibel von 1741, deren Titelkupfer im unteren Viertel den Druckort abbildet, darüber aber biblische Szenen (z.B. Sündenfall, Kain und Abel, Eherne Schlange), die auf die Sündhaftigkeit und Erlösungsbedürftigkeit der Menschen hindeuten (Ba deutsch 1741 01).

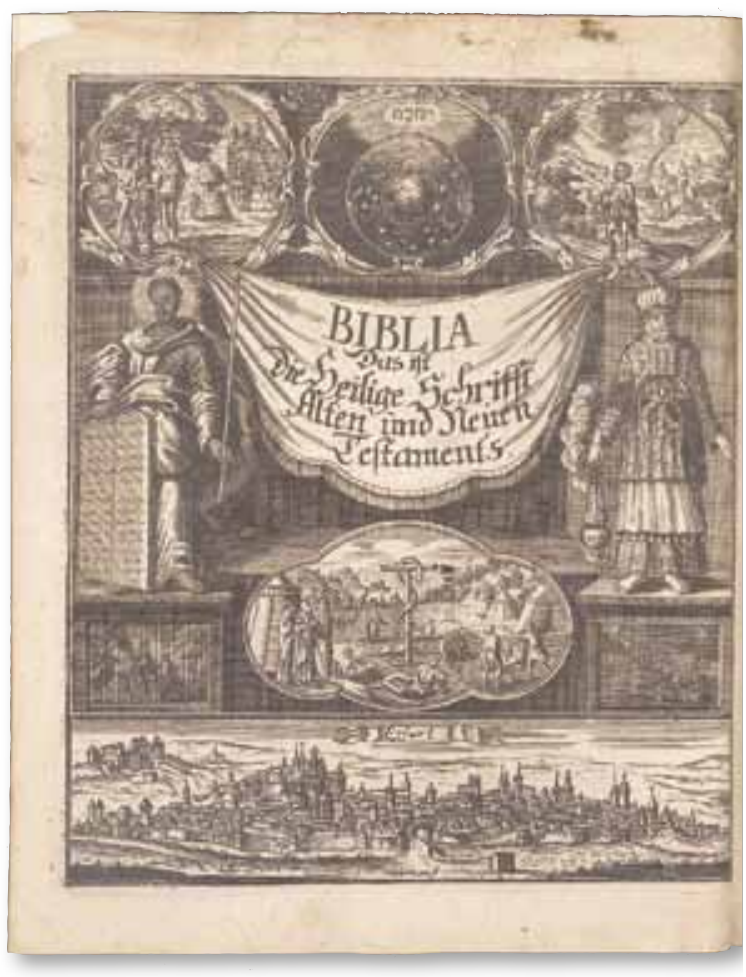

Abb.8: Ba deutsch 174101

Ähnlich verhält es sich mit einer Tübinger Bibel von 1758 (B deutsch 1758 08). Auf dem Titelblatt sieht man unten eine Ansicht von Tübingen, darüber das himmlische Jerusalem. Den Rahmen bilden Szenen zum Spannungsbogen der Heilsgeschichte im Alten und Neuen Testament (Paradies, Sündenfall / Weihnachten, Eherne Schlange / Kreuz Christi, Mose und Paulus). Dies bedeutet: Die vertraute Alltagswelt von Tübingen ist der gegenwärtige Ort des Heilsgeschehens und erweist sich als ambivalente Wirklichkeit mit Sünde und Erlösung. Fast identisch in der Komposition ist eine in den textlichen Beigaben abweichende und in Speyer erschienene Ausgabe von 1767. Die Abbildung Tübingens und des württembergischen Wappens wird - entsprechend dem neuen Adressatenkreis - ersetzt durch eine Ansicht der Stadt Speyer bzw. ihres Doms.

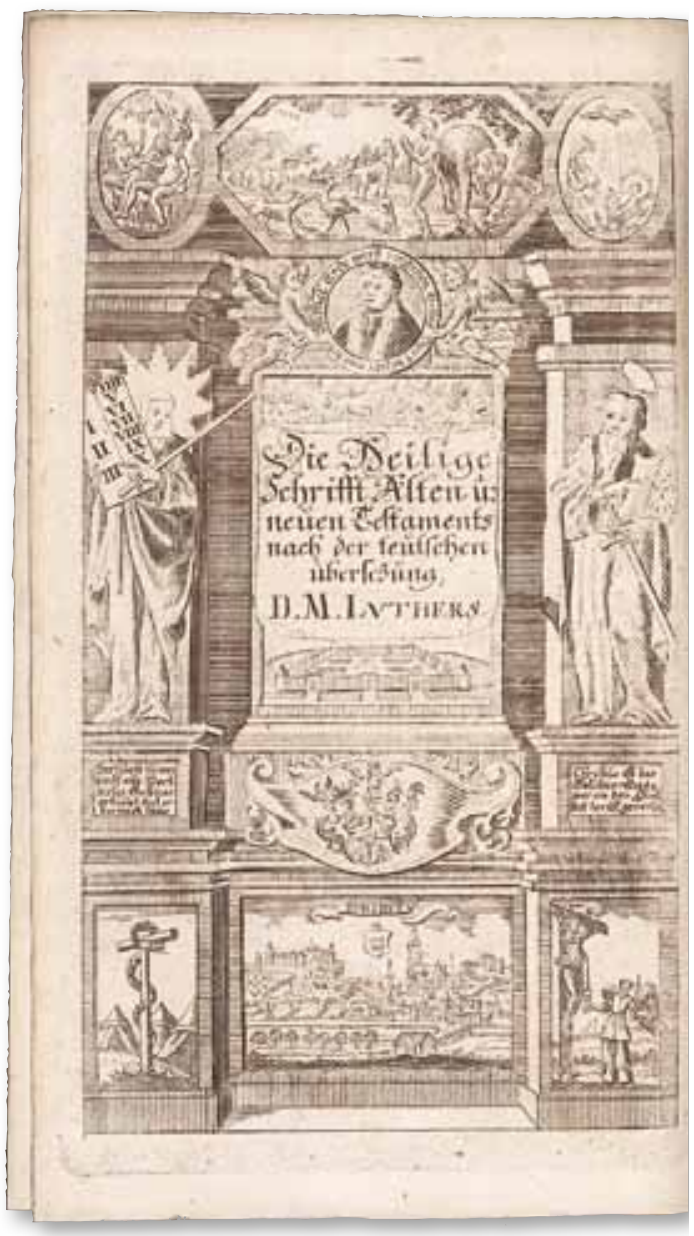

Abb. 9: B deutsch 175808

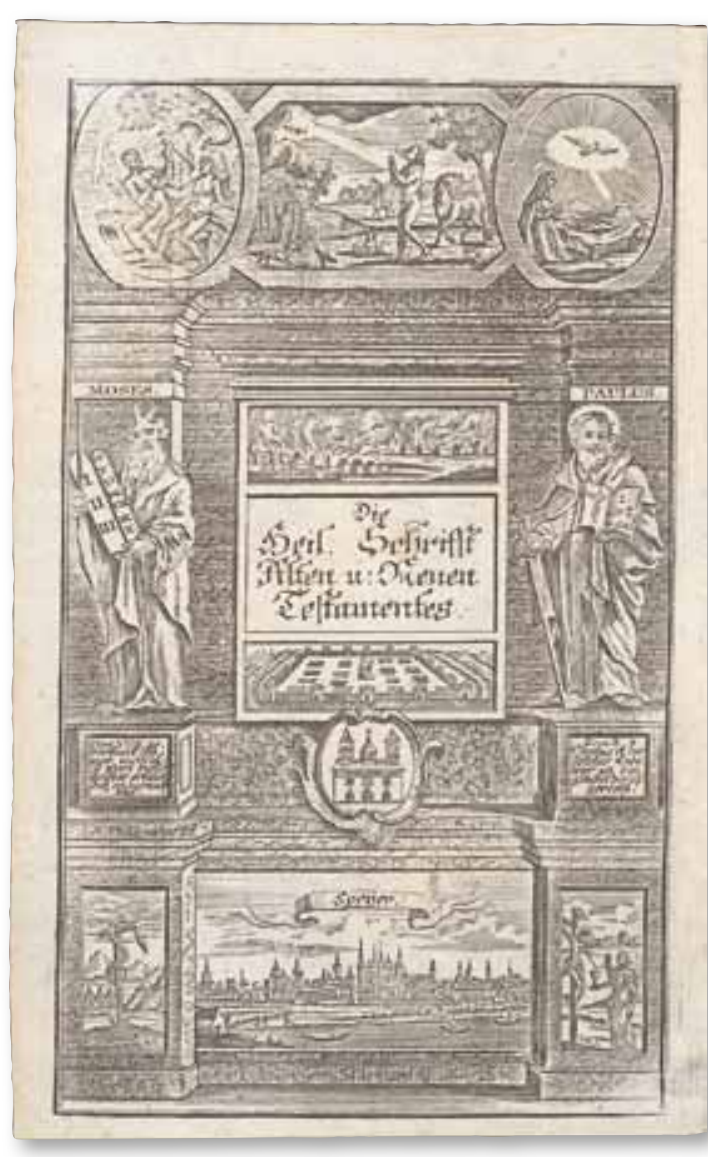

Abb. 10: B deutsch 176701 
Manche Bibeln kombinieren theologische und geographische Motive nicht auf demselben Titelblatt, sondern verteilen die Illustrationen auf mehrere Blätter. Das hat für den Verleger den Vorteil, bei einem anderen Adressatenkreis leichter die spezifischen Stadtansichten austauschen zu können. So begegnen in einer Frankfurter Bibel von 1671 auf dem eigentlichen Titelblatt diverse Szenen aus der biblischen Heilsgeschichte (Bb deutsch 1671 01). Dahinter folgt ein zweiseitiger Kupferstich mit dem Zentrum der Stadt Frankfurt am Main sowie Wappen und Namen der damaligen Ratsmitglieder.

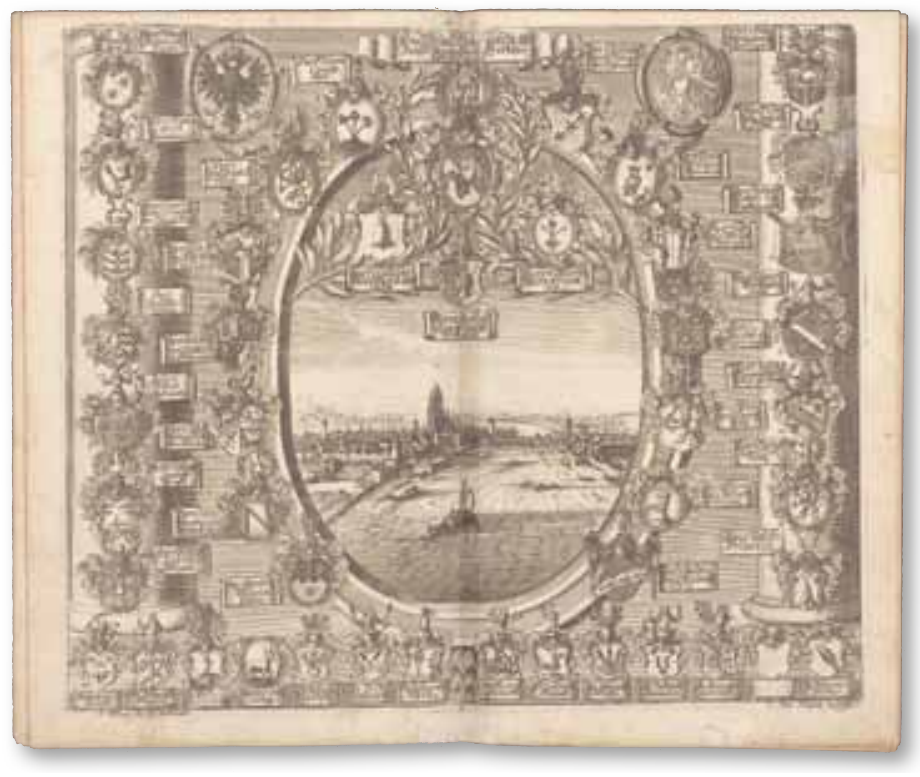

Abb. 11: Bb deutsch 1671 01: Stadtansicht Frankfurts

Mehrfach geschieht eine Anknüpfung an Vertrautes bei tiefer gehender inhaltlicher Füllung in einer Heilbronner Bibel von 1719 (B deutsch 1719 02). Man sieht nicht nur das Stadtbild Heilbronns, sondern mittig den Siebenröhrenbrunnen von 1541, nach dem die Stadt ihren Namen erhielt. Biblische Zitate spielen auf Jesus als Wasser des Lebens (Johannes 4) bzw. auf das Wort Gottes als "Heilsbrunnen" an (Jesaja 12,3). Architektonisch und sprachlich wird angedeutet, dass Heilbronn als Druckort einer Bibel in einem tieferen Sinne dem Bedeutungsgehalt des Ortsnamens entspricht.

Komplex sind auch solche Titelblätter aufgebaut, in denen als weiteres Element Hinweise auf die konfessionelle Prägung hinzukommen. So erkennt man in einer Lutherbibel von 1701 neben theologischen Allegorien und der Stadtansicht von Stade die Büsten Luthers (mit dem Schwan als seinem traditionellen Symbol) und Friedrichs des Weisen,

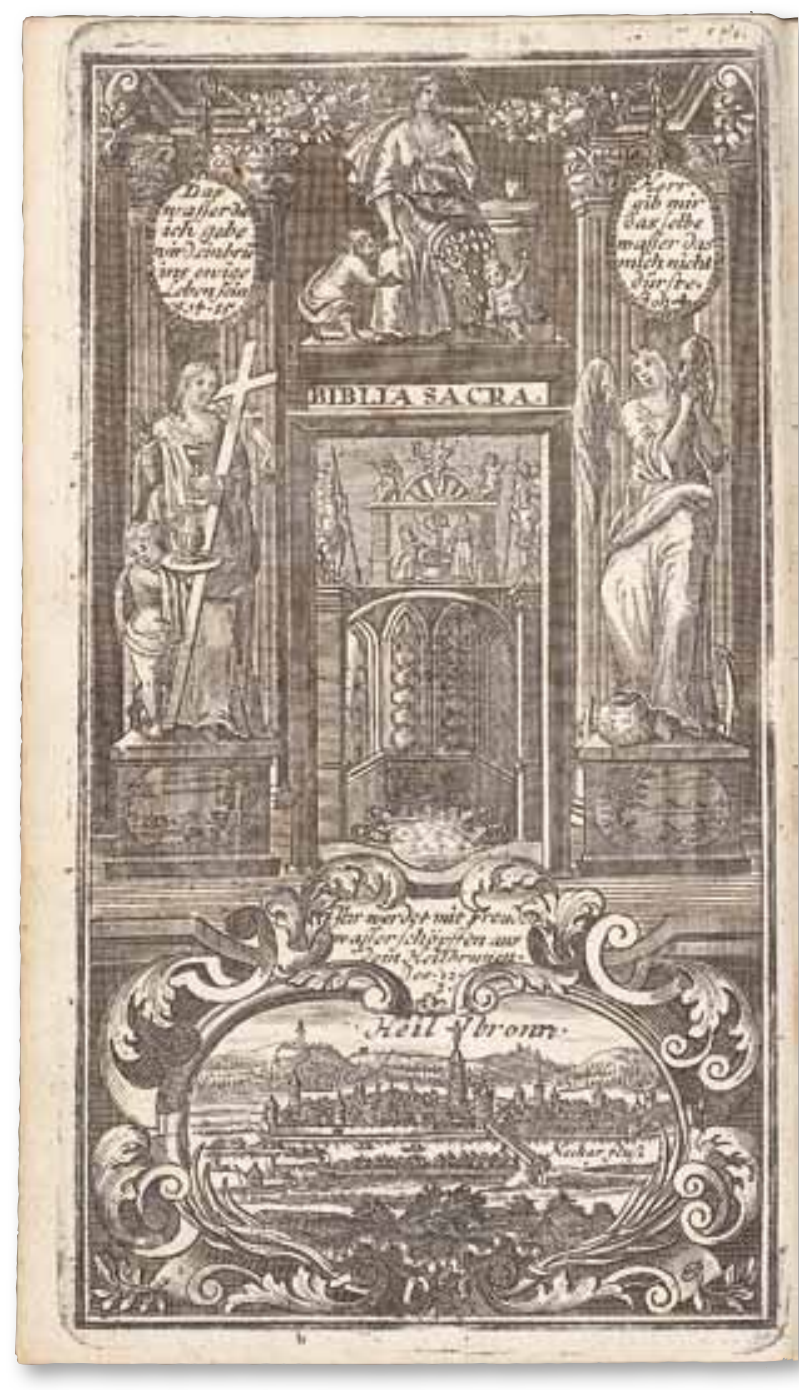

Abb. 12: B deutsch 171902

seines Förderers (mit dem sächsischen Wappen) (Bb deutsch 1701 02).

Charakteristisch für katholische Bibeln ist, dass in den Illustrationen konfessionelle Bezüge wichtiger sind als solche auf die weltliche Obrigkeit. In einer Mainzer Bibel von 1740 erscheint als Gegenüber von Mose die personifizierte Kirche: eine Christusfigur mit Kreuz, Messkelch, Schlüssel für das Petrusamt sowie Papstkrone als Symbol der sakramental verfassten und durch die Ämterstruktur gegliederte Kirche (Bb deutsch 1740 01). Die Evangelistensymbole sind verbunden mit einer von der Kirche bestrahlten Weltkugel, unter der man eine Ansicht von Mainz erkennt. Das Universale (Welt) und Lokale (Mainz) gehört wie Welt- und Ortskirche zusammen. Verbindungsglied ist die Kirche, von der die Bibel, aber auch die Begründung des Staates abhängt. Dementsprechend folgt auf das Titelblatt ein Porträt des amtierenden Fürstbischofs 


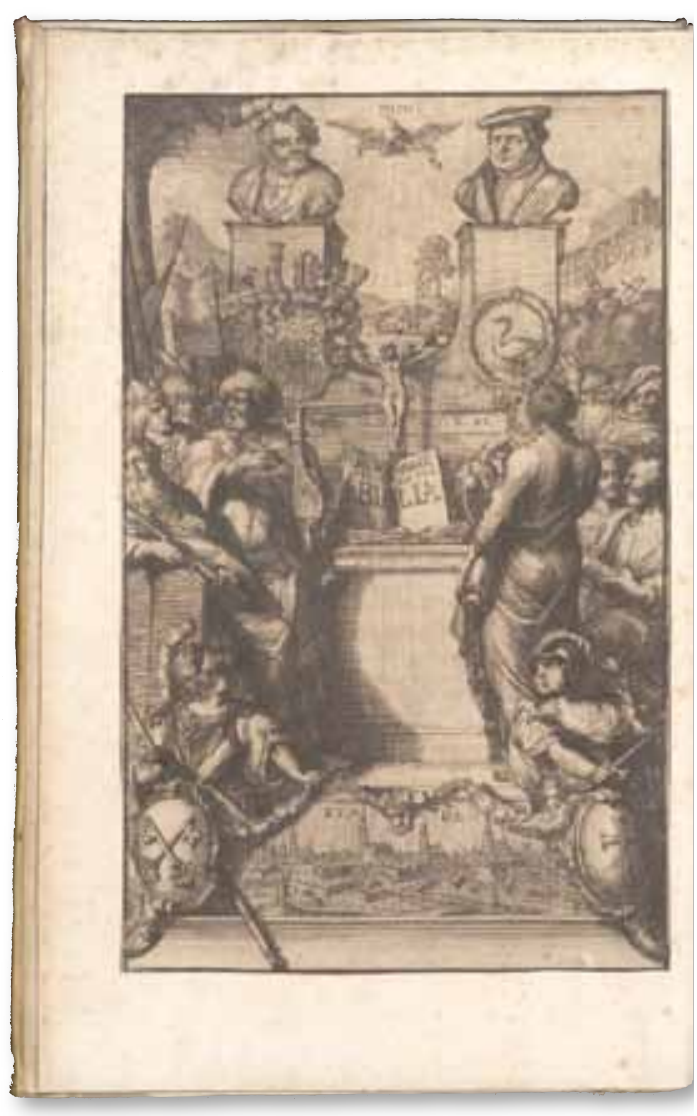

Abb. 13: Bb deutsch 170102

Philipp Karl von Eltz-Kempenich (1665-1743), der nur als geistlicher Herrscher für die Darstellung in einer Bibel akzeptiert werden kann.

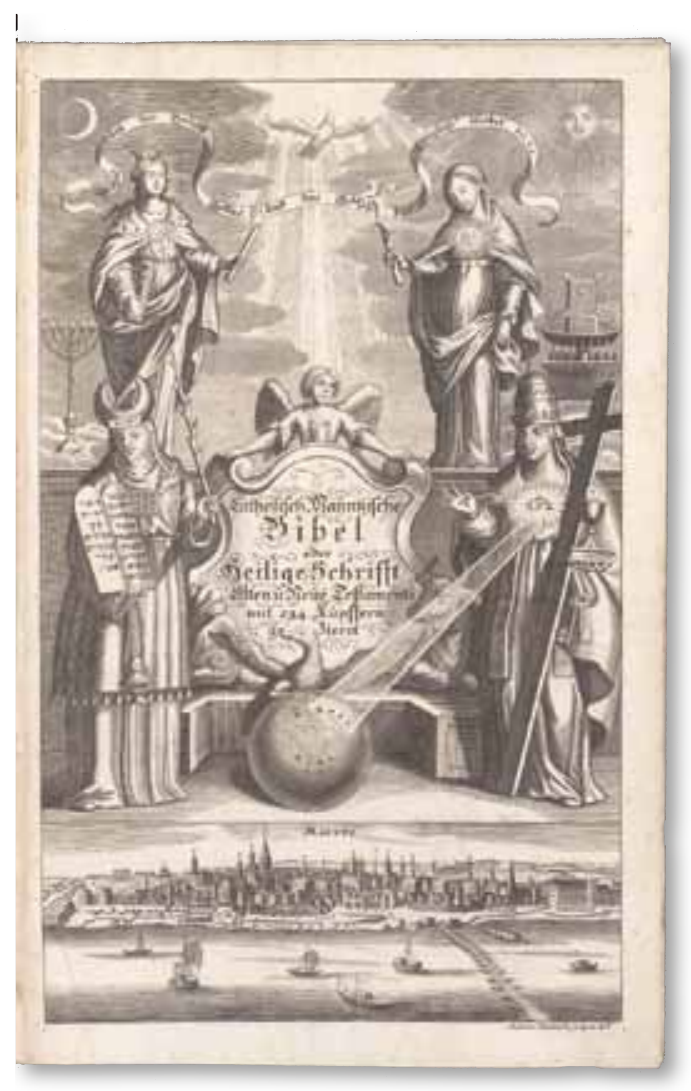

Abb. 14: Bb deutsch 174001
Nicht nur eine geographische, sondern auch sozial-lebensweltspezifische Anknüpfung liegt bei einer 1739 in der Bergbaustadt Freiberg/Sachsen erschienenen Bibel vor (Bb deutsch 1739 01). Auf dem Titelkupferblatt ist das Kreuz Christi vor Freiberg abgebildet, darunter ein Bergwerk mit Abgang und Stollen. Ein Schriftband ("Großer Exegeten fleiß gleicht der Bergleut sauren Schweiß") lädt zum Vergleich zwischen der Bergung von Bodenschätzen und von geistlichen Schätzen in den Tiefen der Heiligen Schrift ein. Zahlreiche Porträtbilder bekannter Schriftausleger werden in Gestalt zweier Obelisken aufgereiht.

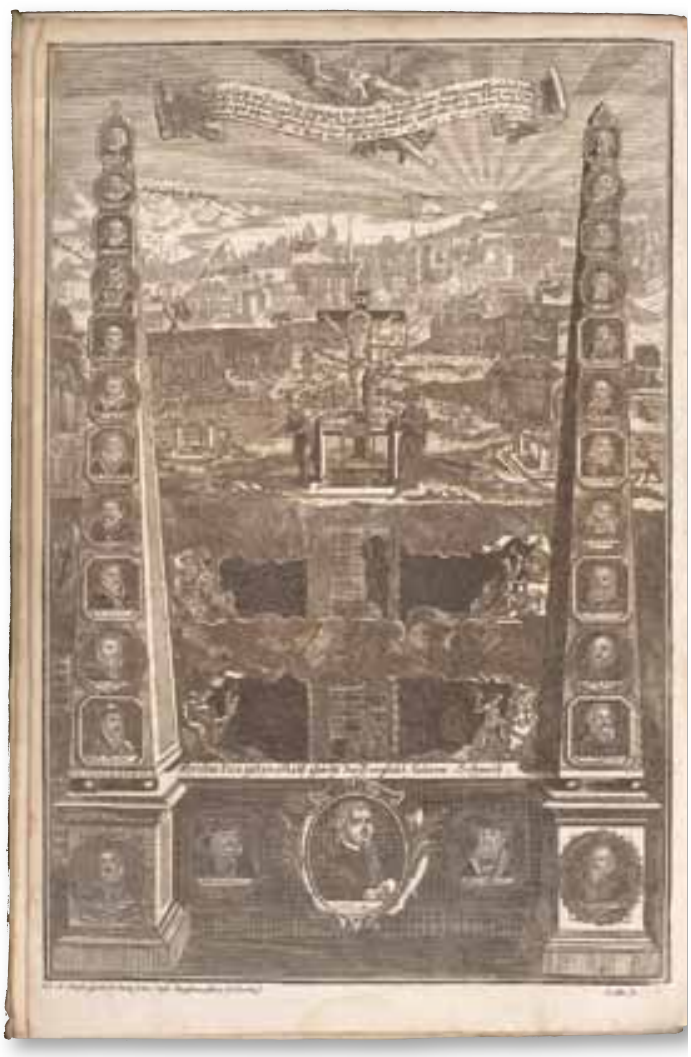

Abb. 15: Bb deutsch 173901

Von der zweiten Hälfte des 18. Jahrhunderts an nimmt der Brauch, Städte in Bibeln abzubilden, wieder ab. Die Kleinfamilie wird wichtiger als die lokal oder territorial definierte Gemeinschaft. Dementsprechend bieten Verleger zunehmend Hausund Familienbibeln an, die mit ihren Beigaben, vor allem Seiten für den Eintrag von Familienereignissen, zur Identitätsbildung von Familien beitragen. Erst im 20. Jahrhundert werden wieder Bibeln mit Abbildungen historisch wichtiger Orte als Hintergrundinformation zur Geschichte der Reformation bzw. zur Umwelt der Bibel angeboten. 